\title{
Accuracy of mortality data for interstitial lung diseases in New Mexico, USA
}

\author{
David B Coultas, Mark P Hughes
}

\begin{abstract}
Background - The sensitivity and accuracy of death certificates and mortality data as sources of population based data on the occurrence of interstitial lung diseases has received limited attention. To determine the usefulness of these data sources, death certificates and mortality data from patients in New Mexico were examined.

Methods-Patients with an interstitial lung disease were identified from a population based registry. For subjects who had died, diagnostic information from their death certificates and from mortality data was compared with the clinical diagnoses made before death.
\end{abstract}

Results - Of 385 patients with a clinical diagnosis of an interstitial lung disease, 134 died between October 1988 and August 1994. Death certificates were obtained for $96 \%$ of these patients. An interstitial lung disease was listed somewhere on the death certificate for only $46 \%$ of the patients, and as an immediate cause of death for only $15 \%$. For the patients with an interstitial lung disease listed somewhere on the death certificate the overall concordance between the diagnoses before death and those on the death certificate was $76 \%$. Mortality data for the State of New Mexico showed a diagnosis of interstitial lung disease to be the assigned cause of death for only $22 \%$ of the patients. The overall agreement between the diagnoses made before death and those of the state mortality data was only $21 \%$.

Conclusions - These results suggest that death certificates and state mortality data are neither sensitive nor accurate for describing the occurrence of interstitial lung diseases. This finding may partly explain the apparently low mortality rates from idiopathic pulmonary fibrosis in the USA compared with other countries.

(Thorax 1996;51:717-720)

Keywords: death certificates, epidemiology, interstitial lung diseases.

Correspondence to: Dr D B Coultas The University of New Mexico School of Medicine, Epidemiology and Cancer Control,

900 Camino de Salud,

Albuquerque, New Mexico

87131, USA.

Received 21 July 1995

Returned to authors

23 October 1995

Revised version received

13 February 1996

Accepted for publication

14 February 1996 eases are not rare, few epidemiological data are available on the occurrence of interstitial lung diseases in the general population. ${ }^{3}$ Death certificates and mortality data are potential sources of population based information on the descriptive epidemiology of interstitial lung diseases. Although the usefulness of these sources of data on interstitial lung diseases has not been well researched, several recent investigations have used mortality data to describe mortality rates for selected interstitial lung diseases. ${ }^{4-6}$

To assess the value of death certificates and state mortality data for describing the occurrence of interstitial lung diseases, we compared diagnostic information from death certificates and state mortality data with clinical diagnoses made before death among a group of patients who had been diagnosed with an interstitial lung disease between 1 October 1988 and 30 September 1992 in Bernalillo County, New Mexico. The patients were identified from a population based registry of interstitial lung diseases established in 1988 to describe the occurrence of these diseases in the county. ${ }^{7}$

\section{Methods}

Several different sources were used to identify patients with interstitial lung diseases aged 18 years and older who were residents of Bernalillo County including pulmonary and primary care physicians, pathology reports, hospital discharge diagnoses, state mortality data, and necropsies. After referral of a potential case of interstitial lung disease to the registry a trained abstractor obtained the medical record to verify the diagnosis. Verification of the diagnosis of an interstitial lung disease required the use of a specific interstitial lung disease term by a clinician caring for the patient. If the clinician used ambiguous language such as "questionable," "possible," or "suggests," or the abstractor had any questions, the chart was also reviewed by one of the authors (DBC). A detailed description of data collection methods for the registry has been described previously. ${ }^{7}$

The vital status of 385 cases identified from the registry was determined by review of medical records and of State of New Mexico mortality data to 1 August 1994. Records of death certificates and state mortality data were obtained for each death. Death certificate data, including all information on the cause of death from Part I(a) to I(d) ("immediate" and three "underlying" causes of death) and Part II ("other significant conditions contributing to death"), were abstracted. Each cause was grouped into one of the following categories: 
Table 1 Distributions of diagnoses listed on death certificates and in the New Mexico mortality data records for patients with interstitial lung diseases (ILD) diagnosed in Bernalillo County, New Mexico who died between 1988 and 1994 $(n=129)$

\begin{tabular}{|c|c|c|c|c|c|}
\hline \multirow[t]{2}{*}{ Diagnosis } & \multicolumn{4}{|c|}{ Death certificates } & \multirow{2}{*}{$\begin{array}{l}\text { State mortality data } \\
\%(n)\end{array}$} \\
\hline & $\begin{array}{l}\text { Listed } \\
\text { anywhere*十 } \\
\%(n)\end{array}$ & $\begin{array}{l}\text { Immediate } \\
\text { cause } \ddagger \\
\%(n)\end{array}$ & $\begin{array}{l}\text { Underlying } \\
\text { cause } S t \\
\%(n)\end{array}$ & $\begin{array}{l}\text { Other significant } \\
\text { conditions } \| t \\
\%(n)\end{array}$ & \\
\hline Respiratory/ILD & $45 \cdot 7(59)$ & $14 \cdot 7(19)$ & $23 \cdot 3(30)$ & $7 \cdot 8(10)$ & $21 \cdot 7(28)$ \\
\hline Respiratory/not ILD & $42.6(55)$ & $31 \cdot 8(41)$ & $11.6(15)$ & $6 \cdot 2(8)$ & $9 \cdot 3(12)$ \\
\hline Cardiovascular & $45 \cdot 7(59)$ & $30 \cdot 2(39)$ & $24 \cdot 8(32)$ & $13 \cdot 2(17)$ & $25.6(33)$ \\
\hline Neoplasms & $19 \cdot 4(25)$ & $13 \cdot 2(17)$ & $6 \cdot 2(8)$ & $1.6(2)$ & $17 \cdot 8(23)$ \\
\hline Gastrointestinal & $7.0(9)$ & $2 \cdot 3(3)$ & $3.9(5)$ & $3 \cdot 1(4)$ & $3 \cdot 1(4)$ \\
\hline Renal disease & $10 \cdot 1(13)$ & $0.8(1)$ & $3.9(5)$ & $5 \cdot 4(7)$ & $1.6(2)$ \\
\hline Accidents & $2 \cdot 3(3)$ & $2 \cdot 3(3)$ & $0.8(1)$ & $0.0(0)$ & $3 \cdot 1(4)$ \\
\hline Other & $21 \cdot 7(28)$ & $4 \cdot 7(6)$ & $8.5(11)$ & $10.9(14)$ & $17 \cdot 8(23)$ \\
\hline
\end{tabular}

*Includes Part I (a)-(d) ("immediate" cause and "underlying" causes) and Part II ("other significant conditions").

+ Total is $>100 \%$ because many death certificates listed $>1$ category of diagnosis.

$\$$ Includes Part I (a) ("immediate cause") only.

f Includes Part I (b)-(d) ("underlying" causes) only.

|| Includes Part II ("other significant conditions") only.

respiratory/interstitial lung diseases, respiratory/not interstitial lung diseases, cardiovascular/cerebrovascular diseases, neoplasms, gastrointestinal diseases, renal diseases, accidents/injuries, and other diseases. If a cause of death was a respiratory/interstitial lung disease, the specific interstitial lung disease diagnosis was also abstracted. Death certificate information was abstracted with no knowledge of the clinical diagnoses before death or the state mortality data codes.

Sensitivity of death certificate and mortality data for detecting interstitial lung diseases was estimated by the proportion of pre-death diagnoses of interstitial lung diseases listed. Diagnostic information on the death certificate was categorised as immediate cause (Part Ia), underlying causes (Part Ib-d), other significant conditions (Part II), and ever listed (Parts Ia-d and II).

The validity of the death certificates and mortality data was determined by comparing the distribution of diagnoses of interstitial lung diseases from these data sources with the distribution of specific diagnoses before death. All analyses were performed using SAS for personal computers. ${ }^{8}$

\section{Results}

Of 385 patients identified with a clinical diagnosis before death of an interstitial lung disease, $134(34.8 \%)$ died between 1 October 1988 and 1 August 1994. Death certificates were located for $129(96.3 \%)$ of the deceased patients, of whom $74(57 \cdot 4 \%)$ were men and for whom the median age at the time of diagnosis was 75 years (range 21-96). The ethnic distribution included $67.4 \%$ non-Hispanic whites, $30.2 \%$ Hispanics, $1.6 \%$ American Indians, and $0.8 \%$ Japanese.

Although interstitial lung diseases, respiratory diseases other than interstitial lung diseases, and cardiovascular diseases were the most common diagnoses listed anywhere on the 129 death certificates (table 1), one or more of these categories was found in less than $50 \%$ of the certificates. In contrast to cardiovascular diseases and other respiratory diseases, interstitial lung diseases were rarely listed as an immediate cause of death $(14 \cdot 7 \%)$ and were more likely to be listed as an underlying cause (23.3\%).

In state mortality data interstitial lung diseases were the second most common cause of death (table 1). However, the sensitivity of mortality data for identifying patients with a clinical diagnosis before death of an interstitial lung disease was only $21 \cdot 7 \%$.

Overall, $55 \%$ of study patients had a diagnosis of pulmonary fibrosis before death (table 2), comprising post-inflammatory pulmonary fibrosis (International Classification of Diseases (ICD)-9 category 515) and idiopathic/ interstitial fibrosis (ICD-9 category $516 \cdot 3$ ). Of these, $71 \cdot 8 \%$ were diagnosed as idiopathic/ interstitial fibrosis. After pulmonary fibrosis, the category of interstitial lung diseases not otherwise specified was the second most frequent diagnosis $(11.6 \%)$, with "other interstitial lung diseases" being far less common.

For all 129 patients with a diagnosis of an interstitial lung disease before death, the diagnoses were infrequently listed on death certificates and state mortality data (table 2). Overall, only $45 \cdot 7 \%(n=59)$ had a diagnosis listed anywhere on the death certificate, and $14.7 \%(n=19)$ had a diagnosis listed as the immediate cause of death. Among all patients with an interstitial lung disease listed on the death certificate, including those listed as the immediate cause of death, the general category of pulmonary fibrosis, comprising post-inflammatory pulmonary fibrosis and idiopathic/ interstitial fibrosis, was listed on $71.2 \%$ and $68.5 \%$ of death certificates, respectively (table $2)$. Within this general category, post-inflammatory pulmonary fibrosis was most frequently listed anywhere or as the immediate cause of death.

A diagnosis before death of an interstitial lung disease was listed as the cause of death in the state mortality data in only $21 \cdot 7 \%(n=28)$ of the cases (table 2). Of these 28 cases, $75 \%$ $(\mathrm{n}=21)$ were included in the general category of pulmonary fibrosis, with $71 \cdot 4 \% \quad(n=20)$ being listed as post-inflammatory pulmonary fibrosis.

From a comparison of diagnoses of interstitial lung diseases from death certificates and state mortality data with those made before death, $76.3 \%$ of the 59 cases $(n=45)$ with an 
Table 2 Distributions of diagnoses of interstitial lung diseases (ILD) listed on medical records, death certificates, and in New Mexico mortality data records for patients with ILD diagnosed in Bernalillo County, New Mexico who died between 1988 and $1994(n=129)$

\begin{tabular}{|c|c|c|c|c|}
\hline \multirow[t]{2}{*}{ Diagnosis (ICD-9 code) } & \multirow{2}{*}{$\begin{array}{l}\text { Medical record } \\
(n=129) \%(n)\end{array}$} & \multicolumn{2}{|l|}{ Death certificate } & \multirow{2}{*}{$\begin{array}{l}\text { State mortality } \\
\text { data } \\
(n=28) \%(n)\end{array}$} \\
\hline & & $\begin{array}{l}\text { Listed anywhere* } \\
(n=59) \%(n)\end{array}$ & $\begin{array}{l}\text { Immediate cause } \dagger \\
(n=19) \%(n)\end{array}$ & \\
\hline Asbestosis (501) & $1.6(2)$ & & & \\
\hline Silicosis $(502)$ & $2 \cdot 3(3)$ & $1 \cdot 7(1)$ & & \\
\hline SLE, lung $(517 \cdot 8,710)$ & $0.8(1)$ & & & \\
\hline Rheumatoid, lung $(517 \cdot 8,714 \cdot 81)$ & $5.4(7)$ & & & \\
\hline Scleroderma, lung $(517 \cdot 8,710 \cdot 1)$ & $2 \cdot 3(3)$ & & & \\
\hline Sjogren's lung $(517 \cdot 8,710 \cdot 2)$ & $0.8(1)$ & $1 \cdot 7(1)$ & & \\
\hline Drug-induced İLD (995·2 + [Ecode]) & $5 \cdot 4(7)$ & $1 \cdot 7(1)$ & & \\
\hline Radiation fibrosis $(508 \cdot 1,995 \cdot 2$ E879.2) & $1.6(2)$ & $3 \cdot 4(2)$ & $5 \cdot 3(1)$ & \\
\hline Goodpasture's syndrome $(446 \cdot 2)$ & $0 \cdot 8(1)$ & $1 \cdot 7(1)$ & & $3 \cdot 6(1)$ \\
\hline Vasculitis $(417 \cdot 8)$ & $0 \cdot 8(1)$ & $1 \cdot 7(1)$ & & \\
\hline Wegener's granulomatosis $(446 \cdot 4)$ & $3 \cdot 1(4)$ & $6 \cdot 8(4)$ & $10 \cdot 5(2)$ & \\
\hline Pulmonary fibrosis $(515,516 \cdot 3)$ & $55 \cdot 0(71)$ & $71 \cdot 2(42)$ & $68 \cdot 5(13)$ & $75 \cdot 0(21)$ \\
\hline Post-inflammatory pulmonary fibrosis (515) & {$[15 \cdot 5](20)$} & {$[44 \cdot 1](26)$} & {$[47 \cdot 4](9)$} & {$[71 \cdot 4](20)$} \\
\hline Idiopathic/interstitial fibrosis $(516 \cdot 3)$ & [39.5] (51) & {$[27 \cdot 1](16)$} & {$[21 \cdot 1](4)$} & {$[3 \cdot 6](1)$} \\
\hline Infectious/post-infectious ILD (011.4) & 0.8 (1) & & & \\
\hline Interstitial pneumonitis & $6 \cdot 2(8)$ & $1 \cdot 7(1)$ & $5 \cdot 3(1)$ & \\
\hline Sarcoidosis $(135,517 \cdot 8)$ & $1.6(2)$ & $1 \cdot 7(1)$ & & $3 \cdot 6(1)$ \\
\hline Diffuse alveolar damage & & $1 \cdot 7(1)$ & $5 \cdot 3(1)$ & \\
\hline ILD, not otherwise specified & $11 \cdot 6(15)$ & $5 \cdot 1(3)$ & $5 \cdot 3(1)$ & $3 \cdot 6(1)$ \\
\hline
\end{tabular}

*Includes Part I (a)-(d) ("immediate" cause and "underlying" causes) and Part II ("other significant conditions") † Includes Part I (a) ("immediate cause") only.

interstitial lung disease listed somewhere on the death certificate matched the diagnoses made before death. However, only $21 \cdot 4 \%$ of the diagnoses in the state mortality data $(n=$ 28) matched the diagnoses before death.

Because pulmonary fibrosis constituted most of the diagnoses of interstitial lung disease made before death (table 2), we used this diagnostic category to examine further the relationship between diagnoses made before death and those listed somewhere on the death certificates or in the state mortality data. Overall, for patients with the general diagnosis of pulmonary fibrosis before death $(n=71)$ and with a diagnosis of an interstitial lung disease on the death certificate $(n=37), 91.9 \%$ had a death certificate diagnosis consistent with pulmonary fibrosis - that is, ICD-9 category $515(n=12)$ or $516.3(n=22)$. However, for the 20 patients with a diagnosis of post-inflammatory pulmonary fibrosis made before death, only $10 \%$ appeared on the death certificate and $5 \%$ in the state mortality data. Although patients with a diagnosis made before death of idiopathic/ interstitial fibrosis were more likely to have this diagnosis listed somewhere on the death certificate $(21.5 \%)$, post-inflammatory pulmonary fibrosis was listed more often somewhere on the death certificate $(39 \cdot 2 \%)$.

Overall, the New Mexico state mortality data diagnoses were consistent with the diagnosis of pulmonary fibrosis made before death $(n=71)$ in $25.4 \%$ of cases. However, in no case was the diagnosis of idiopathic/interstitial fibrosis reported as a cause of death in the state mortality data. Furthermore, the diagnosis of pulmonary fibrosis (categories 515 and 516.3) never appeared in state mortality data without evidence of a clinical diagnosis made before death.

\section{Discussion}

The results of this investigation provide quantitative information on the diagnostic sensitivity and validity of death certificates and state mortality data for describing the occurrence of interstitial lung diseases. We found that both data sources underestimated the occurrence of interstitial lung diseases, and that state mortality data - which are the basis for published mortality rates - missed nearly $80 \%$ of cases, but mortality data never overestimated their occurrence.

Several issues that may influence the interpretation of our findings must be considered. The generally chronic nature of interstitial lung diseases is a factor that may contribute to the underestimation of such cases from mortality data as patients with these diseases may die from causes other than the interstitial lung disease. In a review of five clinical series that included 326 patients who died from idiopathic pulmonary fibrosis, Panos et al ${ }^{9}$ found that 39\% of patients died from respiratory failure due to the underlying lung disease. Because these results were not derived from death certificate or state mortality data, the findings are not directly comparable with the results of our investigation.

Although underestimation of the occurrence of interstitial lung diseases from death certificates and mortality data may be expected, a low concordance between the diagnoses made before death and those on the death certificates and in the mortality data raises additional concerns about the usefulness of mortality data for describing the occurrence of interstitial lung diseases. Because we relied on clinicians' diagnoses of an interstitial lung disease, inaccurate diagnoses made before death may contribute to this low concordance. This is of particular concern for the diagnoses of post-inflammatory pulmonary fibrosis and idiopathic pulmonary fibrosis which may be clinically similar to other interstitial lung diseases and usually require an open lung biopsy for a definitive diagnosis. Because only $7 \%$ of subjects in the New Mexico registry of interstitial lung diseases had an open lung biopsy, ${ }^{7}$ the role of inaccurate clinical diagnoses cannot be assessed in this investigation. Clinical diagnoses obtained by a standardised review of patients' medical records by trained abstractors ${ }^{7}$ represent our best 
available diagnostic information. However, because there was little reclassification of diagnoses, other than between post-inflammatory pulmonary fibrosis and idiopathic/interstitial fibrosis, inaccurate clinical diagnoses cannot explain the substantial diagnostic misclassification in death certificates and mortality data.

A greater use of pulmonary fibrosis compared with idiopathic/interstitial fibrosis on death certificates and in coding mortality data in the USA may partly explain the trends in the nationwide mortality for these two diagnoses since $1979 .{ }^{510}$ Since that date, mortality rates in the USA have been higher for post-inflammatory pulmonary fibrosis than for idiopathic/interstitial fibrosis, and have shown a greater increase.

The infrequent use of the diagnostic term "idiopathic/interstitial fibrosis" in the USA may also contribute to the differences in mortality patterns compared with other countries. Hubbard et $a l^{5}$ have compared mortality rates since 1979 for idiopathic/interstitial fibrosis in seven countries. The mortality rates were highest for England and Wales (rate ratio $=1$ ) and lowest for Germany (rate ratio $=0.087$ ). In contrast, mortality from post-inflammatory pulmonary fibrosis was highest in the USA when compared with other countries. The use of different diagnostic terms with idiopathic pulmonary fibrosis in the USA and cryptogenic fibrosing alveolitis in the UK may partly explain these differences.

In a study published in 1990 Johnston et al determined the sensitivity of death certificates in the UK for detecting idiopathic pulmonary fibrosis among 45 patients with the disease who died during the period 1985-9. They found that $38 \%$ of the death certificates attributed death to idiopathic pulmonary fibrosis, and $56 \%$ had idiopathic pulmonary fibrosis mentioned somewhere on the death certificate. These estimates of sensitivity are higher than in our population (table 2), and suggest that physicians in the UK are more likely to use the specific diagnostic term of idiopathic pulmonary fibrosis on death certificates than physicians in New Mexico.

The results of this investigation suggest that death certificates and mortality data offer little useful information for the study of interstitial lung diseases. However, the sensitivity and validity of these data sources may vary by region within the USA and between countries because of the different patterns in completing death certificates and in coding mortality data. Furthermore, differing patterns of mortality rates in different countries ${ }^{5}$ also suggest that international efforts are needed to standardise the coding of idiopathic/interstitial fibrosis and post-inflammatory pulmonary fibrosis.

This work was supported in part by grant RO1 HL40587 and training grant T32 HL07733, National Heart, Lung and Blood Institute. The authors wish to thank Drs John Britton and Richard Hubbard for their helpful comments, and Rose Whitten, Terry S Davis, and Rita Elliott for assistance in manuscript preparation.

1 Schwarz MI, King TE, eds. Interstitial lung disease. 2nd ed St. Louis, Missouri: Mosby-Year Book, 1993

2 US Department of Health, Education, and Welfare. Respiratory Diseases Task Force. Report on problems, research, approaches, and needs. DHEW Publication No. NIH 76432. Washington, DC: US Government Printing Office: 1972, 1976.

3 Cherniack RM, Crystal RG, Kalica AR. NHLBI Workshop summary. Current concepts in idiopathic pulmonary fib rosis: a road map for the future. Am Rev Respir Dis 1991,
143:680-3.

Johnston I, Britton J, Kinnear W, Logan R. Rising mortality from cryptogenic fibrosing alveolitis. $B M \mathcal{F}$ 1990;301: from crypto 21 .

5 Hubbard RB, Johnston IDA, Coultas DB, Britton JR. Mortality rates from cryptogenic fibrosing alveolitis in seven countries. Thorax 1996;51:711-6.

6 Mannino D, Etzel R. Deaths with idiopathic pulmonary fibrosis in the United States, 1979 through 1989. Am $\mathcal{J}$ Respir Crit Care Med 1994;149:A877.

7 Coultas DB, Zumwalt RE, Black WC, Sobonya RE. The epidemiology of interstitial lung diseases. Am 7 Respir Crit Care Med 1994;150:967-72.

8 SAS Institute. SAS Technical Report P-217, SAS/STAT Software: the PHREG Procedure, Version 6. Cary, North Carolina: SAS Institute, 1991.

9 Panos RJ, Mortenson RL, Niccoli SA, King Jr TE. Clinical deterioration in patients with idiopathic pulmonary fibdeterioration in patients with idiopathic pulmonary fib-
rosis: causes and assessment. Am $\mathcal{F} M e d$ 1990;88:396-404

10 Coultas DB. Epidemiology of idiopathic pulmonary fibrosis. Semin Respir Med 1993;14:181-96. 